\title{
Who needs contacts? Optical fiber poling by induction
}

\author{
D. Huang ${ }^{2}$, F. De Lucia ${ }^{1}$, N. Healy ${ }^{1}$, C. Corbari ${ }^{1}$ and P.J.A. Sazio ${ }^{1 *}$ \\ ${ }^{1}$ Optoelectronics Research Centre, University of Southampton, Southampton, SO17 1BJ, UK \\ ${ }^{2}$ Department of Electrical and Electronic Engineering, Imperial College London, London, SW7 2AZ, UK \\ *pjas@soton.ac.uk
}

\begin{abstract}
Conventional thermal poling methods require direct physical contact to internal fibers electrodes. Here, we report a novel indirect method in which external fields are used to charge floating internal electrodes to generate depletion regions for SHG.

OCIS codes: (190.0190) Nonlinear optics; (190.4370) Nonlinear fibers
\end{abstract}

\section{Introduction}

Since the first reports of the thermal poling of silica fibers almost 25 years ago now, a number of more recent efforts have focused on exploring alternative electrode configurations [1,2] in order to gain both a deeper understanding and to develop more robust methods of device fabrication. In particular, the observation [1] that two internal fiber electrodes at the same positive potential generate a more efficient and stable second order nonlinearity than the conventional positive-negative configuration is at first glance highly counter-intuitive, as the electric field gradient at the midpoint between the two anodes (close to where the poled light guiding core is located) is very small. However, Margulis et al. [1] proposed an "avalanche-like" positive feedback mechanism to explain this poling behaviour, as positive ions migrating away from the anodes immediately lead to a rapid increase in glass resistivity, thus leading to an increase in potential difference at the midpoint. Even more charge movement and larger potential difference between the anodes and fiber midpoint then begins to accumulate until the poling process is completed. Here, we report an indirect poling phenomenon in which it is possible to not only dispense with the cathode, but also for any direct physical contact to the internal anodes themselves.

\section{Experiment}

Silica fibers with an OD of 125 microns and 19 microns internal holes adjacent to a 4.3 micron diameter, 0.17 NA germania doped core, were sourced from Fibercore Ltd, Southampton UK. For our initial induction poling experiments, we used a pressure filling technique similar to the one described by Fokine et al. [3] to fill the internal holes with BiSn eutectic alloy as shown in Fig. 1 (left). However, in order to minimize optical absorption losses it is advantageous to remove the metal after poling and therefore in all subsequent experiments, Gallium was used as the liquid conductor as it can easily be removed by re-pressurizing the fiber.
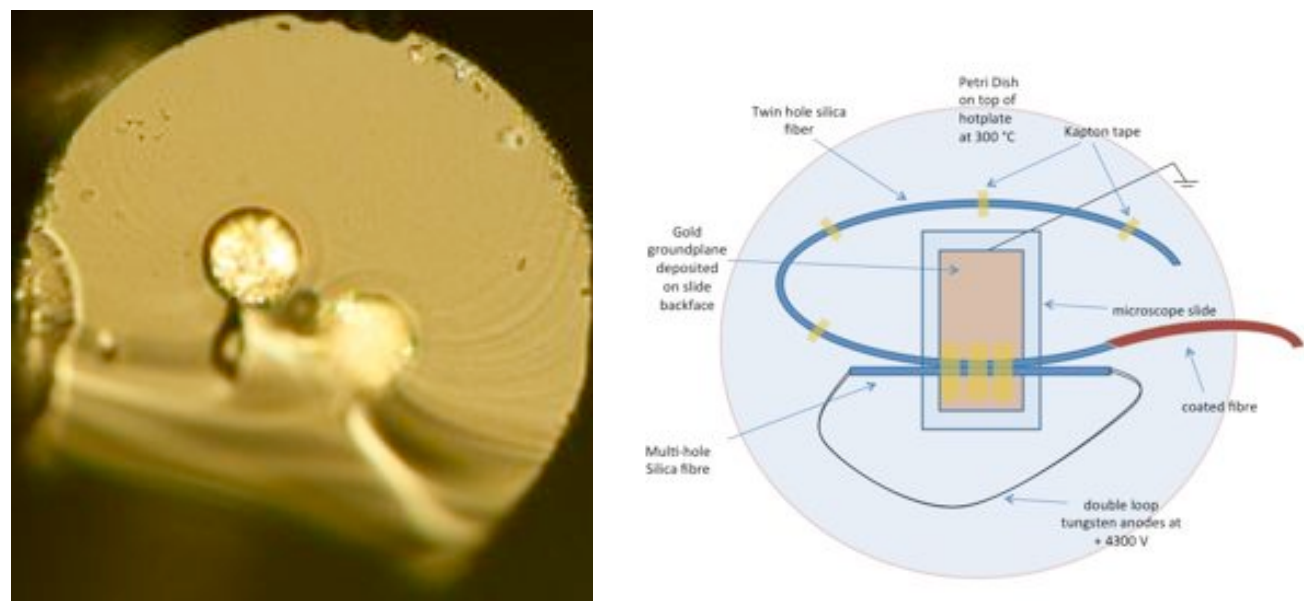

Fig.1. (Left) Twin 19 micron holes in silica fiber with a germania doped core. The depletion regions generated by induction poling are visible after a HF decoration etch and can be seen surrounding the internal BiSn electrodes. (Right) The induction poling setup with inductor and sample in close proximity. Both are uncoated, although the sample remains attached to the original coated section outside the hot zone .

The induction poling setup itself consists of two separate fibers with embedded electrodes, namely the 'inductor' and the sample. As shown in Fig. 1 (right), the inductor consists of a $6 \mathrm{~cm}$ length of uncoated multi-hole silica 
capillary with two 18 micron diameter tungsten wires manually inserted inside. Both wires form a long double loop that is connected to a HV source, typically operated at $+4300 \mathrm{~V}$. The sample is mounted immediately adjacent to the inductor and both are adhered onto a standard microscope slide that has an evaporated gold layer on the opposite face, used as a grounding backplane. The sample can be much longer that the inductor itself and in fact as far as we are aware, there is no length restriction so long as the sample maintains the correct poling temperature. The slide, sample and inductor are placed into a glass petri dish on a hotplate with a temperature of $300^{\circ} \mathrm{C}$. The positive voltage is then applied to the inductor and the sample is left to pole for 120 mins before the heater is switched off and allowed to cool with the HV source on for about 60 mins.

The depletion regions seen in Fig.1 (left) are typical and representative of those observed throughout the entire $20 \mathrm{~cm}$ length of the induction poled sample after (destructive) sectioning and etching. We make the reasonable assumption that the induction mechanism operates by "charging up" the electrically floating BiSn or Gallium wires inside the sample. To model this behaviour, we have used COMSOL Multiphysics numerical simulations of the electric potential distributions between the inductor, the sample and the ground plane. Fig. 2 (left) shows a 2D map of the equipotentials calculated using Gauss' law before charged ion migration begins to generate the depletion regions. A line scan of the electric potential along $\mathrm{x}=0$ through the common geometric centres of the two fibers as seen in Fig. 2 (right) shows the large variation in electric field within the sample being poled, thus strongly suggesting that an enhanced "avalanche-like" positive feedback mechanism [1] plays an important role in the induction poling technique. We will discuss both the simulations and optical characterization of the induction poled fibers, along with analysis of SHG output.

Finally we note that this novel poling technique lifts a number of restrictions on the use of complex microstructured optical fibers for poling, as it is no longer necessary to individually contact wires inside. Selective liquid metal filling both radially and longitudinally can then allow for indirect poling inside any complex geometry.
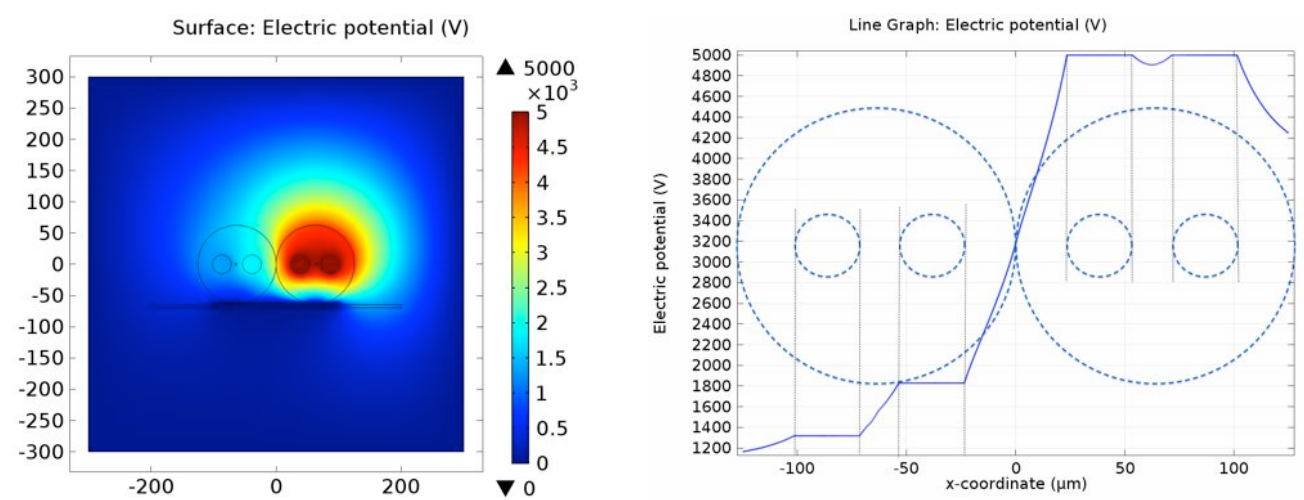

Fig.2. (Left) 2D electric potential distribution. A $+5 \mathrm{kV}$ potential is applied to both anodes of the inductor, whilst both wires inside the sample are left floating. (Right) A line scan through the geometric centres of the fibers reveals large potential variations in the sample that can drive impurity ion migration for depletion formation.

\section{Acknowledgements}

The authors acknowledge EPSRC EP/I035307/1 and C. Corbari acknowledges the EU project CHARMING (contract No. FP7-288786) for financial support.

\section{References}

[1] W. Margulis, O. Tarasenko and N. Myren, "Who needs a cathode? Creating a second order nonlinearity by charging glass fiber with two anodes", Opt. Exp. 17, 15534-15540 (2009).

[2] H. An and S. Fleming, "Investigating the effectiveness of thermally poling optical fibers with various internal electrode configurations", Opt. Exp. 20, 7436-7444 (2012).

[3] M. Fokine, L.E. Nilsson, A.A. Claesson, D. Berlemont, L. Kjellberg, L. Krummenacher and W. Margulis, "Integrated fiber Mach-Zehnder interferometer for electro-optic switching", Opt. Lett. 27, 1643-1645 (2002). 